\title{
La Educación y la Prevención como Componentes Primordiales en la Actitud hacia las Políticas de Drogas
}

\section{Education And Prevention as Principal Components in Attitudes Toward Drugs Policies}

\author{
Wilson López-López \\ Claudia Pineda-Marín \\ Pontificia Universidad Javeriana. Bogotá - Colombia \\ Etienne Mullet \\ Ecole Practique des Hautes Études. Toulouse, Francia
}

(Rec: 03 de agosto de 2012 / Acept: 01 de octubre de 2012)

\begin{abstract}
Resumen
Las políticas para el manejo de las drogas en el mundo han sido principalmente prohibicionistas, han tenido un alto costo social, político y económico y pobres resultados en la disminución de la producción, el tráfico y el consumo de drogas. Este estudio piloto indagó la aceptabilidad frente a diversas políticas de drogas de 100 ciudadanos y ciudadanas corrientes de Bogotá-Colombia muestreados aleatoriamente. Para recoger los datos se usó un instrumento de 12 escenarios con dos conceptos fundamentales: el compromiso del Estado con la prevención y educación, y los tipos de políticas de los Estados. Los resultados se obtuvieron con análisis de varianzas y un análisis de componentes principales. Encontramos que las personas tienen un mayor nivel de aceptabilidad hacia las políticas de reglamentación, menor aceptabilidad hacia las políticas de libre mercado y que las personas tienen mayor aceptabilidad cuando el Estado se compromete con campañas de educación y prevención.

Palabras clave: legalización, reglamentación, libre mercado, prevención, reducción de daños.
\end{abstract}

\begin{abstract}
Drugs policies around the world have been mainly prohibitionists. It have had a high social, political and economic price and poor results in decreasing production, trafficking and drugs consumption. In this pilot research, we studied the level of acceptability of various drugs policies, among 100 ordinary people, men and women, sampled randomly. To collect data we used 12 scenarios with two fundamental concepts: State's commitment with prevention and education and drugs policies. The results were extracted by variance analysis and a principal component analysis. We found that people had a major level of acceptability with regulatory policies than of free market policies. Moreover people had a major level of acceptability when the State was commitment with education and prevention.

Keywords: legalization, drugs regulation, free market, prevention, harm reduction.
\end{abstract}

Correspondencia: Dr. Wilson López-López, Pontificia Universidad Javeriana, Facultad de Psicología, Dirección Carrera 5 No 39-00 Edificio 95-Manuel Briceño S.J., Bogotá - Colombia. E-mail: lopezw@javeriana.edu.co 


\section{Introducción}

Aproximadamente 50 años atrás en Estados Unidos germinaba uno de los futuros problemas que Colombia y otros países latinoamericanos habrían de afrontar: "la lucha antidrogas", con todos los costos humanos, económicos y políticos que ésta ha representado. Mientras por un lado se declaraba la represión contra el consumo de drogas como la marihuana, los $\mathrm{LSD}^{1}$ y la heroína, paradójicamente por otro lado la cocaína era considerada una sustancia de baja peligrosidad, apta para consumidores glamurosos, ejecutivos de negocios, estrellas del cine y de la música (Gaviria y Mejía, 2011; Gaviria et al., sf; Mejía y Caballero, 2012). El precio de la lucha antidrogas parece ser más letal que el consumo clandestino de las sustancias psicoactivas, puesto que las organizaciones que controlan el negocio han emprendido guerras que dejan más de 30.000 muertos al año, además han infiltrado y cooptado las instituciones gubernamentales, sin contar que gracias al negocio han financiado su arsenal y esta lucha le cuesta a Colombia por lo menos 10.000 millones de dólares al año (Mejía, 2011).

Aunque se han ido dando transformaciones en cuanto a las políticas para el manejo de las drogas en el mundo, Latinoamérica aún sigue siendo un territorio prohibicionista, pero esta política no ha sido más que un fracaso; es entonces cuando algunos sectores de la academia comenzamos a preguntarnos cuáles podrían ser las estrategias alternativas para disminuir los daños causados por las políticas de drogas y por el abuso de las mismas. En ese sentido, este artículo presenta un estudio piloto en el que se encuestaron ciudadanos y ciudadanas ordinarios, para conocer su nivel de aceptabilidad con respecto a diversas políticas de drogas y la relación entre esas políticas y su percepción de la necesidad de que el Estado no solo se comprometa a controlar y corregir sus políticas, sino a implementar programas de prevención y cuidado de la salud asociados al consumo de las drogas.

Para dar contexto al estudio aquí realizado, es importante puntualizar que las políticas de tipo prohibicionista han tenido consecuencias que no se esperaban y además están soportadas en discursos circulantes que no corresponden de manera precisa con la realidad que muestran algunos informes emitidos por instituciones gubernamentales, no gubernamentales e investigaciones académicas. Hay 5 consecuencias identificadas por la Comisión Global de Políticas de Drogas (CGPD) en su informe de 2011; a saber: el crecimiento del mercado negro criminal, el desplazamiento político (cooptación del Estado), el desplazamiento geográfico (o efecto globo), el desplazamiento de sustancias (cambio del tipo de sustancia consumida) y la estigmatización, marginación y exclusión de los consumidores (Calvani, 2006; Thoumi, 2006).

Dietilamida de ácido lisérgico.
Sobre los discursos que sostienen las políticas prohibicionistas se tienen registros muy antiguos, en los que las drogas fueron calificadas como sustancias malditas, diabólicas y asociadas al paganismo (que se enfrentaba al cristianismo); así, la represión se sustentaba en creencias religiosas, racistas y moralistas (Majón-Cabeza, 2012). Además discursos tales como que si se legaliza la droga incrementarán la producción, los consumidores, la delincuencia y las conductas pecaminosas son algunos que hoy día se mantienen entre los sectores más conservadores de las sociedades, pero estudios como el de Camacho, Gaviria y Rodríguez (2011) muestran que a pesar de la prohibición, la cantidad de consumidores incrementó entre 1996 y 2008 en Colombia, en todos los niveles socioeconómicos y en todas las regiones del país. Además Mejía (2011) encontró que realmente los más afectados con el denominado "garrote ${ }^{2 "}$ eran los campesinos productores de la hoja de coca, quienes a su vez obtienen menos ganancias del negocio.

En octubre de 2010, en el marco de la fallida legalización de la marihuana en California, la Revista Semana publicó que la legalización le ahorraría a Estados Unidos 13.500 millones de dólares que gasta en la lucha contra las drogas y podría generar 106 millones de dólares en impuestos por la administración del negocio de las drogas; también expusieron que en México, la legalización de las drogas lograría disminuir notablemente el desempleo y facturar hasta 20.000 millones de dólares en ventas.

Esto no quiere decir que la legalización sin ningún tipo de regulación sería la política más apropiada, puesto que una política de libre mercado podría resultar tan peligrosa como la prohibición, sobre todo para el consumidor, a quien nadie le asegura la calidad del producto que consume y la probabilidad del daño en contra de su salud sería alta. A diferencia de estos dos extremos, Suiza, Inglaterra y los Países Bajos son un ejemplo de reglamentación de las drogas con resultados bastante positivos. En Suiza se implementó un programa de sustitución de la heroína cuyos efectos se reflejaron en la reducción del consumo, los niveles de actividad criminal asociados al negocio y el alejamiento entre los consumidores ocasionales y sus expendedores (CGPD, 2011).

En Inglaterra se implementó un programa de derivación de las prisiones a centros de tratamiento para el consumo de las drogas, cuyo impacto fue la reducción de los delitos en un $48 \%$, de acuerdo con los informes de años anteriores a la implementación del programa. En los Países Bajos, las investigaciones muestran que el porcentaje de personas que consumen heroína no incrementa aunque no hay prohibición. En este último ejemplo, se hace evidente que drogas como la heroína disminuyeron su demanda debido a que las campañas de educación y prevención mostraron

\footnotetext{
2 "Garrote" hace referencia a los castigos aplicados a quienes cultivan la coca.
} 
que era altamente adictiva. Además, el suministro de jeringas, espacios controlados para el consumo e incluso el abastecimiento de la droga en las cantidades apropiadas hicieron que los daños en contra de la salud y los delitos menores se redujeran (CGPD, 2011; Pali, Greenfield \& Reuter, 2012).

Esto último hace pensar que el componente educación y prevención tiene una incidencia fundamental en la actitud hacia las políticas de regulación, pues si se considera que además de la prohibición y el libre mercado podrían aplicarse otras políticas alternativas con resultados positivos ya demostrados en otros países, la flexibilidad en los países latinoamericanos tal vez pueda aumentar y en esa medida los gobernantes no perciban que al afirmar que están de acuerdo con nuevas políticas son considerados pecadores o antiéticos (y viceversa, los que se oponen parezcan más decorosos). Entre las propuestas del Informe Nacional de Desarrollo Humano (2003), se hace hincapié en que la reducción de los daños tiene que ser una prioridad tanto a nivel individual (en el caso de los consumidores) como ambiental (eliminando prácticas de fumigación aérea), y para ello nada mejor que el compromiso del Estado con la implementación de políticas más realistas que la eliminación total de los consumidores, tales como la educación, la prevención y la rehabilitación (Nadelmann, 2006).

Para recoger los datos de esta investigación y analizar los resultados nos basamos en la Teoría de Integración de la Información (Anderson, 2008), en la que se propone fundamentalmente que las personas tomamos los estímulos del ambiente, les damos una valoración, integramos esos estímulos (siguiendo un heurístico de suma, multiplicación o promedio), luego tenemos una representación psicológica y finalmente emitimos una respuesta (juicio o acción). Con esta misma teoría se han desarrollado otros estudios en diversos lugares del mundo y abordado temas de interés social tales como el perdón (Bagnulo, Muñoz-Sastre \& Mullet, 2009), la eutanasia (Mas, Albaret, Sorum \& Mullet, 2010), la ruptura de la confidencialidad (Ollivari, MuñozSastre, Guedj, Clay-Sorum \& Mullet, 2011), el aborto (Muñoz- Sastre, Pecarisi, Legrain, Mullet, \& Sorum, 2007), la legitimidad de las intervenciones militares, las amnistías políticas (Kpanake \& Mullet, 2011), temas que en esencia comprometen los juicios de las personas.

De acuerdo con lo anterior, en este estudio indagamos qué dice la gente sobre la legalización, la prohibición y la regulación de las drogas, y cuáles son las reglas cognitivas que inciden en sus juicios. Es claro que el conocimiento que se genera sobre fenómenos sociales de alto impacto -como éste- en general no emerge de lo que expresa la gente de la calle, más bien escuchamos a los expertos, políticos, economistas, juristas, científicos sociales y a los psicólogos -estos últimos hablando sobre todo de tratamientos-, todos con intereses diversos y convergentes pero que podrían enriquecerse más si se integran con las actitudes y las expectativas que tiene la gente que no se dedica a estudiar estos temas.

\section{Método}

\section{Diseño}

Para este estudio se usó una metodología cuantitativa y un diseño factorial no experimental, posteriormente los datos se analizaron descriptivamente y se compararon las medias entre las características de cada escenario, y finalmente se hizo un análisis de componentes principales.

\section{Instrumento}

El instrumento usado en esta investigación tuvo un diseño ortogonal 2 x 6: Prevención por parte del Estado $x$ Política de drogas. En todos los escenarios se le propuso al participante un país hipotético productor de drogas; en la mitad de los escenarios el Estado realizaba acciones de tipo educativo y preventivo sobre el consumo de drogas y en la otra mitad, el Estado no se involucraba en acciones concretas ni de educación ni de prevención. Los escenarios combinaron 6 tipos posibles de políticas de Estado, la primera fue el libre mercado de todas las drogas, la segunda fue la prohibición de todas las drogas, la tercera fue la reglamentación de todas las drogas, la cuarta fue la reglamentación de las drogas blandas y la prohibición de las duras, la quinta fue el libre mercado de las drogas blandas y la prohibición de las duras, y la sexta y última fue el libre mercado de las drogas blandas y reglamentación de las duras.

Un ejemplo de un escenario es el siguiente: Mirza es un pequeño país de 10 millones de habitantes. En Mirza, como en los países vecinos, hay mucho consumo interno tanto de las drogas blandas (marihuana) como de las drogas duras (cocaína, heroína, LSD). Este país también es productor de las drogas. Muchas áreas rurales de este país se utilizan en el cultivo y la producción de las drogas. Algunos de los cultivos y los laboratorios existen de manera clandestina, camuflados entre otros cultivos o escondidos en lugares recónditos del país. En este Estado no se han hecho campañas de información veraz para advertir a las personas sobre los peligros asociados con el consumo de drogas. En este país el gobierno ha prohibido completamente tanto la producción como el consumo y el mercadeo de las drogas duras (cocaína, heroína, LSD), además de ello ha decidido judicializar a quienes se involucren en ese negocio; $\sin$ embargo, el gobierno de Mirza ha decidido no inmiscuirse, ni a favor ni en contra del mercado de las drogas blandas (marihuana).En Mirza, las drogas duras se venden de manera ilegal; el precio de las drogas es bastante alto, no tiene límites y se han generado condiciones de corrupción que llegan a infiltrar incluso altos niveles del gobierno y las instituciones públicas, adicional a eso los homicidios 
han aumentado por la lucha entre carteles. Por otra parte, las drogas blandas (marihuana) no tienen ningún tipo de regulación ni vigilancia. El Estado no controla el precio de las drogas blandas, que en este país es bastante alto pero no tanto como para que las personas no puedan tener acceso a ellas. En cuanto a la calidad de las drogas, se puede decir que es variable, hay drogas de buena calidad y otras de mala calidad, al punto que algunas han llegado a generar accidentes mortales (por sobredosis accidentales).

El instrumento preguntaba ¿qué tan de acuerdo está usted con la política con la que manejan las drogas en ese Estado? y luego el participante marcaba en una escala Osgood -que tenía valores entre 1 y 10 , siendo 1 nada de acuerdo y 10 completamente de acuerdo- su nivel de acuerdo con el escenario propuesto.

\section{Participantes}

El presente estudio se llevó a cabo en Bogotá- Colombia y contó con la participación de 100 personas, ciudadanos y ciudadanas comunes, de edades entre los 18 y 55 años, de los niveles socio-económicos 2, 3, 4, 5 y 6 . El muestreo fue de tipo probabilístico, específicamente un muestreo aleatorio estratificado y proporcional. Otro de los criterios para seleccionar a los participantes fue su voluntad para participar. Para asegurar el nivel socio-económico ${ }^{3}$, los instrumentos fueron aplicados en la casa de cada uno de los participantes.

\section{Procedimiento}

Cada uno de los participantes firmó un consentimiento informado y luego de las instrucciones sobre cómo responder y llenar la escala, el participante contestó 4 escenarios de familiarización con el tipo de instrumento. Los escenarios de familiarización no fueron tenidos en cuenta para los análisis, solo tuvieron la intención de entrenar la forma de responder el cuadernillo principal.

Después de completar el ejercicio de familiarización, cada participante comenzó a leer escenario por escenario y a marcar su nivel de aceptación; contestar a cada cuadernillo tardó entre 30 y 45 minutos. Es importante señalar que los participantes accedieron a contestar el cuadernillo de manera voluntaria, sin recibir ningún pago por hacerlo.

\section{Resultados y discusión}

Los resultados más importantes se muestran en la Figura 1 y la Tabla 1. En la Tabla 1 se muestran los resultados de la prueba ANOVA de cada una de las políticas y la comparación entre las medias de cada política y si cada una fue implementada con campañas de educación y prevención.

En Colombia, los niveles socio-económicos corresponden a la diferenciación entre clases sociales donde 1 es la más baja y 6 es la clase más alta.
La P1 hace referencia a la política de libre mercado de las drogas, la P2 se refiere a la política de prohibición de todas las drogas, $\mathrm{P} 3$ a la política de libre mercado de las drogas blandas y prohibición de las drogas duras, $\mathrm{P} 4$ a la reglamentación de drogas blandas y prohibición de las duras, P5 a la reglamentación y P6 al libre mercado de drogas blandas y reglamentación de drogas duras. En la Figura 1 se observa, en el eje vertical, el nivel de aceptabilidad de las políticas de drogas de los participantes (siendo 1 nada de acuerdo y 10 completamente de acuerdo). En el eje horizontal se pueden ver las medias de los diferentes tipos de políticas planteados en los escenarios, y finalmente cada una de las líneas muestra las medias de aceptabilidad dependiendo del

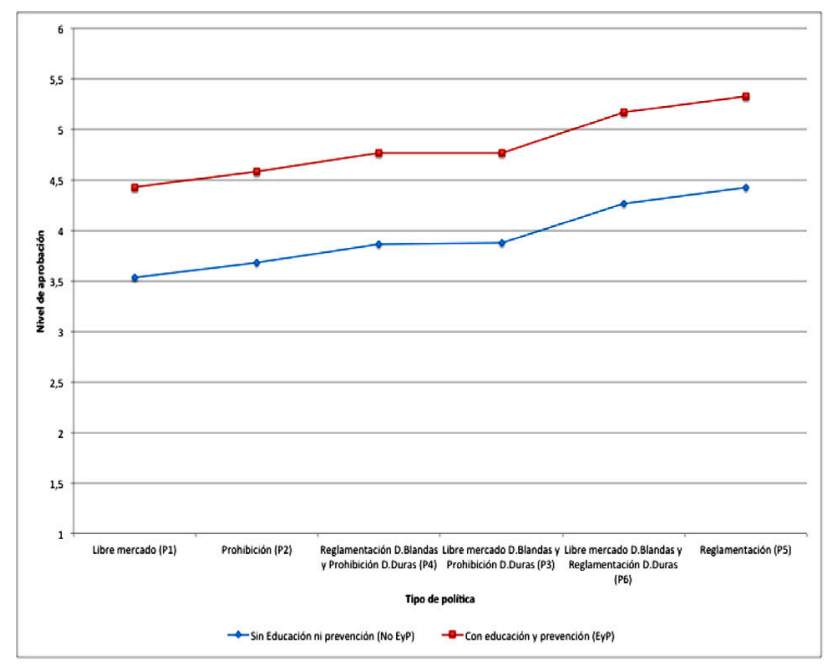

Figura 1. Aceptabilidad de las políticas de drogas en relación con la educación y prevención sobre el consumo de drogas.

compromiso que el gobierno tiene de implementar programas de educación y prevención del consumo de drogas.

En estos resultados se resalta que la aceptabilidad de los escenarios propuestos fue media-baja $(M=4.39$, en una escala 0 a 10). Además, es evidente y estadísticamente significativa la diferencia en la aceptación de cada una de las políticas cuando los escenarios incluyeron un componente de educación y prevención a cuando no lo tuvieron (ver tabla 1).

De acuerdo con la figura 1, la menor aceptación $(M=3.53)$ se registra cuando el escenario propone una política de libre mercado y no hay compromiso del Estado (literalmente el escenario propone: (...) En este Estado no se han hecho campañas de información, ni de prevención para advertir a las personas sobre los peligros asociados con el consumo de drogas. En este país el gobierno no quiere encargarse de regular ni la producción, ni el consumo, ni el tráfico de las drogas, en otras palabras el gobierno de Mirza ha decidido no inmiscuirse, ni a favor ni en contra del mercado de las drogas (...)).

El segundo lugar de menor a mayor aceptación lo ocupan las políticas prohibicionistas $(M=3.68)$, que en los escenarios 
Tabla 1: ANOVA de políticas de drogas en relación con el compromiso del Estado (educación y prevención).

\begin{tabular}{|c|c|c|c|c|c|c|c|}
\hline Política & $\begin{array}{c}\text { Compromiso del } \\
\text { Estado }\end{array}$ & & SS & $\mathrm{gl}$ & SM & $\mathrm{F}$ & Sig. \\
\hline \multirow[t]{6}{*}{$\mathrm{P} 1$} & EyP & Inter-grupos & 443.555 & 14 & 31.682 & 18.491 & .00 \\
\hline & & Intra-grupos & 145.64 & 85 & 1.713 & & \\
\hline & & Total & 589.194 & 99 & & & \\
\hline & No EyP & Inter-grupos & 101.305 & 14 & 7.236 & 4.339 & .00 \\
\hline & & Intra-grupos & 141.744 & 85 & 1.668 & & \\
\hline & & Total & 243.049 & 99 & & & \\
\hline \multirow[t]{6}{*}{$\mathrm{P} 2$} & EyP & Inter-grupos & 336.08 & 16 & 21.005 & 6.888 & .00 \\
\hline & & Intra-grupos & 253.114 & 83 & 3.05 & & \\
\hline & & Total & 589.194 & 99 & & & \\
\hline & No EyP & Inter-grupos & 127.459 & 16 & 7.966 & 5.72 & .00 \\
\hline & & Intra-grupos & 115.59 & 83 & 1.393 & & \\
\hline & & Total & 243.049 & 99 & & & \\
\hline \multirow[t]{6}{*}{ P3 } & EyP & Inter-grupos & 358.465 & 15 & 23.898 & 8.7 & .00 \\
\hline & & Intra-grupos & 230.73 & 84 & 2.747 & & \\
\hline & & Total & 589.194 & 99 & & & \\
\hline & No EyP & Inter-grupos & 107.872 & 15 & 7.191 & 4.469 & .00 \\
\hline & & Intra-grupos & 135.177 & 84 & 1.609 & & \\
\hline & & Total & 243.049 & 99 & & & \\
\hline \multirow[t]{6}{*}{$\mathrm{P} 4$} & EyP & Inter-grupos & 444.168 & 16 & 27.76 & 15.888 & .00 \\
\hline & & Intra-grupos & 145.027 & 83 & 1.747 & & \\
\hline & & Total & 589.194 & 99 & & & \\
\hline & No EyP & Inter-grupos & 156.655 & 16 & 9.791 & 9.406 & .00 \\
\hline & & Intra-grupos & 86.393 & 83 & 1.041 & & \\
\hline & & Total & 243.049 & 99 & & & \\
\hline \multirow[t]{6}{*}{ P5 } & EyP & Inter-grupos & 368.1 & 16 & 23.006 & 8.637 & .00 \\
\hline & & Intra-grupos & 221.094 & 83 & 2.664 & & \\
\hline & & Total & 589.194 & 99 & & & \\
\hline & No EyP & Inter-grupos & 105.069 & 16 & 6.567 & 3.95 & .00 \\
\hline & & Intra-grupos & 137.98 & 83 & 1.662 & & \\
\hline & & Total & 243.049 & 99 & & & \\
\hline \multirow[t]{6}{*}{ P6 } & EyP & Inter-grupos & 381.799 & 15 & 25.453 & 10.309 & .00 \\
\hline & & Intra-grupos & 207.395 & 84 & 2.469 & & \\
\hline & & Total & 589.194 & 99 & & & \\
\hline & No EyP & Inter-grupos & 137.604 & 15 & 9.174 & 7.308 & .00 \\
\hline & & Intra-grupos & 105.445 & 84 & 1.255 & & \\
\hline & & Total & 243.049 & 99 & & & \\
\hline
\end{tabular}

Nota. En la tabla EyP hace referencia a los escenarios en los que hay compromiso de educación y prevención por parte del Estado; No EyP hace referencia a los escenarios en los que el compromiso del Estado no incluía ni educación ni prevención. 
fueron propuestas así: (...) En este país el gobierno ha prohibido completamente tanto la producción, como el consumo y el mercadeo de las drogas blandas (marihuana) y las drogas duras (cocaína, heroina, LSD), además de ello ha decidido judicializar a quienes se involucren en ese negocio (...).

En tercer lugar -de menor a mayor aceptación- se ubicaron tanto los escenarios que mostraban políticas de reglamentación de drogas blandas y prohibición de drogas duras (P4), como los escenarios de las políticas de libre mercado de las drogas blandas y prohibición de las drogas duras (P3). Tanto en el caso en que el Estado ofrecía educación y prevención $(M=5.02$ para P3 y $M=4.82$ para P4) como en el caso que no $(M=4.12$ para P3 y $M=3.91$ para P4), no tuvieron diferencias estadísticamente significativas $(t(100,99)=1.93, p>.05$ en la comparación entre P3 y P4 con educación y prevención; $t(100,99)=1.93, p>.05$ en la comparación entre P3 y P4 sin educación ni prevención).

En el cuarto lugar -de menor a mayor aceptación-, la política de libre mercado de las drogas blandas y reglamentación de las drogas duras (P6) obtiene una $M=4.27$ para cuando el Estado no se compromete con educación ni prevención. Literalmente el escenario decía: El Estado se ha encargado de regular tanto la producción como el consumo y el mercadeo de las drogas duras (cocaina, heroina, LSD), como de las drogas blandas (marihuana). El gobierno de Mirza vende las drogas duras (cocaína, heroina, LSD) correctamente empacadas, en dosis apropiadas y se expenden en tiendas que son vigiladas y reguladas por el gobierno. Los costos para poder hacer parte del negocio de las drogas son tan altos que los traficantes tuvieron que abandonar este mercado. Por otra parte, las drogas blandas (marihuana) no tienen ningún tipo de regulación ni vigilancia. El Estado no controla el precio de las drogas blandas, que en este país es bastante alto pero no tanto como para que las personas no puedan tener acceso a ellas.

Finalmente, la política de reglamentación (P5) resulta tener mayor aceptación que todas las anteriores $(M=4.43$ cuando no hay educación ni prevención y $M=5.33$ cuando se propone educación y prevención), literalmente estos escenarios proponían: El Estado se ha encargado de regular tanto la producción como el consumo y el mercadeo de las drogas duras, como el mercado de las drogas blandas.

De acuerdo con los datos recogidos se realizó un análisis de componentes principales en el que se identificaron 3 componentes que explican el $71.05 \%$ de la varianza (ver tabla 2). Como se ve en la tabla 2, el componente 1 explica el $43.89 \%$ de la varianza total, el componente 2 explica el $15.56 \%$ de la varianza y el componente 3 explica el 11.60 $\%$ de la varianza total. De acuerdo con la prueba KMO, se encontró que los patrones de correlación fueron relativamente compactos, en ese sentido los componentes hallados fueron confiables $(K M O=.80)$, de igual manera la prueba de esfericidad tuvo un $p=.00$. En cuanto a los componentes hallados, la tabla 3 muestra la matriz de componentes rotados, en los que se ve que el componente 1 agrupa las tendencias de las respuestas de los escenarios 1 y 19 al 24; el componente 2 agrupa las tendencias de las respuestas en los escenarios 2, 4 y 5 ; finalmente, el tercer grupo estuvo conformado por las tendencias de las respuestas en los escenarios 3 y 6 . En la tabla 3 también se muestra el elemento común de cada uno de los grupos de respuestas.

De acuerdo con estos hallazgos iniciales del estudio, se pueden identificar tres aspectos fundamentales. En primer lugar es evidente que el componente educación y

Tabla 2. Análisis de la varianza de los componentes principales.

\begin{tabular}{|c|c|c|c|c|c|c|c|c|c|}
\hline \multirow[t]{2}{*}{ Componente } & \multicolumn{3}{|c|}{ Autovalores iniciales } & \multicolumn{3}{|c|}{$\begin{array}{l}\text { Suma de las saturaciones al } \\
\text { cuadrado de la extracción }\end{array}$} & \multicolumn{3}{|c|}{$\begin{array}{l}\text { Suma de las saturaciones al } \\
\text { cuadrado de la rotación }\end{array}$} \\
\hline & Total & $\begin{array}{l}\% \text { de la } \\
\text { varianza }\end{array}$ & $\%$ acumulado & Total & $\begin{array}{l}\% \text { de la } \\
\text { varianza }\end{array}$ & $\%$ acumulado & Total & $\begin{array}{l}\% \text { de la } \\
\text { varianza }\end{array}$ & $\%$ acumulado \\
\hline 1 & 5.266 & 43.879 & 43.879 & 5.266 & 43.879 & 43.879 & 4.341 & 36.176 & 36.176 \\
\hline 2 & 1.868 & 15.564 & 59.443 & 1.868 & 15.564 & 59.443 & 2.277 & 18.974 & 55.15 \\
\hline 3 & 1.393 & 11.606 & 71.05 & 1.393 & 11.606 & 71.05 & 1.908 & 15.899 & 71.05 \\
\hline 4 & 0.832 & 6.937 & 77.986 & & & & & & \\
\hline 5 & 0.671 & 5.592 & 83.579 & & & & & & \\
\hline 6 & 0.524 & 4.363 & 87.941 & & & & & & \\
\hline 7 & 0.321 & 2.678 & 90.619 & & & & & & \\
\hline 8 & 0.303 & 2.521 & 93.14 & & & & & & \\
\hline 9 & 0.273 & 2.277 & 95.418 & & & & & & \\
\hline 10 & 0.22 & 1.837 & 97.254 & & & & & & \\
\hline 11 & 0.198 & 1.647 & 98.901 & & & & & & \\
\hline 12 & 0.132 & 1.099 & 100 & & & & & & \\
\hline
\end{tabular}


Tabla 3. Matriz de componentes rotados y características de cada escenario.

\begin{tabular}{|c|c|c|c|c|c|c|}
\hline \multirow[t]{2}{*}{ Escenario } & \multicolumn{3}{|c|}{ Componente } & \multicolumn{2}{|c|}{ Características del escenario } & \multirow{3}{*}{$\begin{array}{c}\begin{array}{c}\text { Característica de } \\
\text { agrupación }\end{array} \\
\text { Tendencias explica- } \\
\text { das mayormente por } \\
\text { el compromiso del } \\
\text { Estado }\end{array}$} \\
\hline & 1 & 2 & 3 & $\begin{array}{l}\text { Compromiso del } \\
\text { Estado }\end{array}$ & $\begin{array}{l}\text { Tipo de política de manejo de las } \\
\text { drogas }\end{array}$ & \\
\hline E19 & .87 & & & $\begin{array}{l}\text { Con educación y } \\
\text { prevención }\end{array}$ & Libre mercado de todas las drogas & \\
\hline E23 & .85 & & & $\begin{array}{l}\text { Con educación y } \\
\text { prevención }\end{array}$ & $\begin{array}{l}\text { Reglamentación de las blandas y } \\
\text { prohibición de las duras }\end{array}$ & \\
\hline E22 & .82 & & & $\begin{array}{l}\text { Con educación y } \\
\text { prevención }\end{array}$ & Reglamentación de todas las drogas & \\
\hline E21 & .78 & & & $\begin{array}{l}\text { Con educación y } \\
\text { prevención }\end{array}$ & $\begin{array}{c}\text { Libre mercado de las drogas blandas } \\
\text { y prohibición de las duras }\end{array}$ & \\
\hline E24 & .75 & & & $\begin{array}{l}\text { Con educación y } \\
\text { prevención }\end{array}$ & Prohibición de todas las drogas & \\
\hline E20 & .68 & & & $\begin{array}{l}\text { Con educación y } \\
\text { prevención }\end{array}$ & $\begin{array}{l}\text { Libre mercado de las drogas blandas } \\
\text { y reglamentación de las duras }\end{array}$ & \\
\hline E1 & .51 & & & $\begin{array}{l}\text { Sin educación ni } \\
\text { prevención }\end{array}$ & Libre mercado de todas las drogas & \\
\hline E4 & & .88 & & $\begin{array}{l}\text { Sin educación ni } \\
\text { prevención }\end{array}$ & Reglamentación de todas las drogas & $\begin{array}{l}\text { Tendencias expli- } \\
\text { cadas mayormente }\end{array}$ \\
\hline E5 & & .83 & & $\begin{array}{l}\text { Sin educación ni } \\
\text { prevención }\end{array}$ & $\begin{array}{l}\text { Reglamentación de las blandas y } \\
\text { prohibición de las duras }\end{array}$ & $\begin{array}{l}\text { por las políticas } \\
\text { que proponen la }\end{array}$ \\
\hline E2 & & .56 & & $\begin{array}{l}\text { Sin educación ni } \\
\text { prevención }\end{array}$ & $\begin{array}{l}\text { Libre mercado de las drogas blandas } \\
\text { y reglamentación de las duras }\end{array}$ & reglamentación \\
\hline E3 & & & .84 & $\begin{array}{l}\text { Sin educación ni } \\
\text { prevención }\end{array}$ & $\begin{array}{c}\text { Libre mercado de las drogas blandas } \\
\text { y prohibición de las duras }\end{array}$ & $\begin{array}{l}\text { Tendencias explica- } \\
\text { das mayormente por }\end{array}$ \\
\hline E6 & & & .81 & $\begin{array}{l}\text { Sin educación ni } \\
\text { prevención }\end{array}$ & Prohibición de todas las drogas & $\begin{array}{l}\text { la prohibición de las } \\
\text { drogas duras }\end{array}$ \\
\hline
\end{tabular}

prevención tiene incidencia en la actitud de las personas frente a la aceptabilidad, puesto que se considera que en condiciones de mayor información y generación de espacios recreativos diferentes al consumo de drogas, la probabilidad de que una persona se haga consumidor habitual es menor. En estudios similares que se han realizado con el consumo de alcohol se encontró que las personas consideran alternativas de atención y acompañamiento diferentes a la mera prohibición o denuncia ante las autoridades, tales como la rehabilitación, la participación en grupos de apoyo, el acompañamiento de profesionales, la ayuda de los familiares y la orientación (Salazar, Valdez, Martínez \& Pedroza, 2011).

Por otro lado y de acuerdo con la teoría de integración de la información propuesta por Norman Anderson (2008), en este caso los resultados coinciden con la denominada ley de la suma, en la que los componentes de un escenario se suman para dar como resultado la actitud, en otras palabras, Componente educación y prevención + Componente
Política = Aceptabilidad de las políticas de drogas; la actitud frente a las políticas de drogas es el resultado de un procesamiento cognitivo en el que los estímulos se integran de manera aditiva.

En segundo lugar, las personas tienen mayores niveles de aceptabilidad hacia las políticas de reglamentación. Aunque no es claro cuáles son las razones por las que consideran que ésta puede ser otra opción, es presumible que las personas entrevistadas compartan el hecho de que las políticas prohibicionistas no han tenido resultados exitosos en cuanto a la reducción del consumo o los homicidios relacionados con las guerras entre carteles. Por el contrario, el Estado sigue sin comprometerse concretamente con la reducción de daños. Estos puntajes contrastan con los obtenidos en las políticas de libre mercado y prohibición, que como se dijo al inicio pueden tener graves consecuencias en los consumidores, puesto que no se garantiza la calidad de los productos que se consumen y los riesgos en la salud son bastante altos (CGPD, 2011). 
En tercer y último lugar, los resultados señalan que hay tres componentes principales que indican que aún puede haber personas que no estén muy seguras de cuál sea la política más apropiada y con mejores resultados para el manejo de la droga, y hay gran responsabilidad de los medios de comunicación en este aspecto, puesto que la oportunidad para llevar a cabo debates serios y basados en evidencias es reducido, por el contrario, se le da prelación a las opiniones apoyadas en los prejuicios y creencias de figuras públicas que aunque pueden ser puntos de referencia en otros temas de Estado, no lo son en políticas de manejo de drogas.

Evidentemente este es un estudio que comienza a explorar un aspecto de las políticas que antes no había sido muy indagado, y es la actitud de la gente común frente a problemáticas sociales donde ellos mismos son protagonistas o víctimas. Dicho esto, la indagación con respecto al tema y las acciones concretas para el éxito de una política alternativa de manejo de las drogas no puede ser menos que revisada minuciosamente, considerando las consecuencias de la implementación de programas a gran escala y las limitaciones que ello tiene, pero definitivamente teniendo presente que la historia ya nos sirve como indicador de los errores en las intervenciones antidroga, sus vacíos y las alternativas de educación, control y prevención que pueden resultar efectivas.

Para el futuro se espera que este estudio pueda ser realizado con una muestra más grande en la que los resultados mostrados aquí puedan precisarse y de igual manera se puedan describir las características demográficas (sexo, edad, nivel socio-económico, religión, nivel educativo) de quienes participan en el estudio y sus tendencias en las respuestas a los escenarios propuestos. Esto último a propósito del reciente estudio de Vargas y Trujillo (2012), quienes muestran que las tendencias en el consumo de cannabis ha incrementado en los grupos de mujeres jóvenes y cómo algunas variables relacionadas con la composición familiar y la educación de los padres (De la Villa Moral \& Ovejero, 2011) se constituyen como factores de riesgo en la prevalencia del consumo y la iniciación del mismo. Sugerimos que en una próxima investigación se indaguen los hábitos de consumo de sustancias de los participantes, puesto que es presumible que al ser consumidor de alguna sustancia ilegal esto tenga incidencia en su actitud y además haya diferencias significativas con los participantes que consumen alguna sustancia legal como cigarrillo o alcohol y los que no consumen ninguna sustancia.

\section{Referencias}

Anderson, N. (2008). Unified social cognition. New York: Psychology Press Taylor \& Francis Group, LLC.

Bagnulo, A., Muñoz-Sastre, M.T., \& Mullet, E. (2009). Concepciones de perdón: Una comparación entre Latinoamérica y Europa. Universitas Psychologica, 8, 673-682.
Camacho, A., Gaviria, A., \& Rodríguez, K. (2011). El consumo de drogas en Colombia. En A. Gaviria \& D. Mejía (Eds.), Política antidroga en Colombia: éxitos, fracasos y extravios (pp. 41-65). Bogotá: Uniandes.

Calvani, S. (2006). ¿Debería Colombia reducir aún más sus cultivos ilícitos, o al contrario, debería reducir el número de productores de cocaína, esclavos de los grupos armados? En A. Camacho (Ed.), Narcotráfico: Europa, Estados Unidos, América Latina (pp. 3-12). Bogotá: Uniandes - Ceso.

Comisión Global de Políticas de Drogas. (Junio de 2011). Guerra a las drogas. Extraído el 1 de junio de 2012. Disponible en http://www.drogasextremadura.com/archivos/Global_Commission_Report_Spanish.pdf

De la Villa Moral, M., \& Ovejero, A. (2011). Consumo abusivo de alcohol en adolescentes españoles: tendencias emergentes y percepciones de riesgo. Universitas Psychologica, 10, 71-87.

Gaviria, A., \& Mejía, D. (2011). Políticas antidroga en Colombia: Éxitos, fracasos y extravios. Bogotá: Ediciones Uniandes.

Gaviria, C., Zedillo, E., Cardoso, F., Romero, A.M., Mockus, A., García, D., Krauze, E., Santos, E., Cardoso, A., Marinho, J., Vargas, M., Naím, M., Llerena, P., Cohelo, P., Ramírez, S., Picado, S., y Martínez, T. (S.F.). Drogas y democracia: Hacia un cambio de paradigma. Declaración de la Comisión Latinoamericana sobre Drogas y Democracia. Recuperado el 1 de marzo de 2011 de http://www.drogasedemocracia.org/Arquivos/ declaracao_espanhol_site.pdf

Kpanake, L., \& Mullet, E. (2011). Judging the acceptability of amnesties. Conflict Resolution Quarterly, 28, 291-313.

Majón-Cabeza, A. (2012). La solución. Bogotá: Debate.

Mas, C., Albaret, M. C., Sorum, P. C., \& Mullet, E. (2010). French general practitioners vary in their attitudes toward treating terminally-ill patients. Palliative Medicine, 24, 60-67.

Mejía, D., \& Caballero, C. (2012). Narcotráfico y lavado de activos: La dimensión del problema. En Notas de Politica. 11. Extraído el 1 de junio de 2012. Disponible en http://gobierno.uniandes.edu.co/Publicaciones/ Notas $\% 20 \mathrm{de} \% 20$ Politica/notas $\% 20 \mathrm{de} \% 20$ politica11-internet.pdf

Mejía, D. (2011). Política antidroga en el Plan Colombia: Costos, efectividad y eficiencia. En A. Gaviria \& D. Mejía (Eds.), Política antidroga en Colombia: Éxitos, fracasos y extravios (pp. 69-90). Bogotá: Editorial Uniandes.

Muñoz-Sastre, M. T., Pecarisi, C., Legrain, E., Mullet, E., \& Sorum, P. C. (2007). Acceptability in France of induced abortion for adolescents. American Journal of Bioethics, 7, 26-32.

Nadelmann, E. (2006). La reducción de los daños de la prohibición de las drogas en las Américas. En A. Camacho (Ed.), Narcotráfico: Europa, Estados Unidos, América Latina (pp. 63-76). Bogotá: Uniandes - Ceso.

Ollivari, C., Muñoz-Sastre, M.T., Guedj, M., Clay-Sorum, P., \& Mullet, E. (2011). La ruptura de la confidencialidad: una comparación entre las perspectivas chilena y francesa respecto a las condiciones de su aceptabilidad. Universitas psychologica, 10,13-26.

Pali, L., Greenfield, V., \& Reuter, P. (2012). Change is possible: The history of the international drug control regime and implications for future policymaking. Substance Use \& Misuse, 47, 923-035.

Revista Semana (octubre de 2010). ¿Marihuana legal?. Disponible en http://www.semana.com/nacion/marihuana-legal/146636-3.aspx

Salazar, G. M. L., Valdez, R. J. F., Martínez, M. K. I., \& Pedroza, C. F. J. (2011). Intervenciones breves con adolescentes estudiantes rurales que consumen alcohol en exceso. Universitas Psychologica, 10, 803-815.

Vargas, C., \& Trujillo, H. M. (2012). Cannabis consumption by female Psychology students: The influence of perceived stress, coping and consumption of drugs in their social environment. Universitas Psychologica, 11, 119- 130.

Thoumi, F. (2006). Las políticas contra las drogas, reformas y relaciones colombo-americanas. En A. Camacho (Ed.), Narcotráfico: Europa, Estados Unidos, América Latina (pp. 163-184). Bogotá: Uniandes - Ceso. Informe Nacional de Desarrollo Humano Colombia. (2003). El conflicto, callejón con salida. Bogotá: PENUD. 